\title{
Modelling Renal Filtration and Reabsorption Processes in a Human Glomerulus and Proximal Tubule Microphysiological System
}

\author{
Stephanie Zhang ${ }^{1}$ and Gretchen Mahler ${ }^{2}$ \\ ${ }^{1}$ SUNY Binghamton \\ ${ }^{2}$ Binghamton University
}

April 5, 2021

\begin{abstract}
Kidney microphysiological systems (MPS) serve as valuable preclinical instruments in recapitulating physiological conditions and determining underlying interactions involved in renal clearance and osmoregulation. Current kidney MPS models target individual regions of the nephron, such as the glomerulus and proximal tubule, but fail to incorporate multiple filtration and absorption interfaces. In the current study, an in vitro MPS features key filtration and reabsorption properties of the human glomerulus and proximal tubule for seven days of operation. Three human-derived cell types, including the conditionally immortalized human podocytes (CIHP-1), human umbilical vein endothelial cells (HUVECs), and human proximal tubule cells (HK-2), were adapted to serum-free medium prior to being seeded into the three-component MPS (T-Junction splitter, glomerular housing unit, and parallel proximal tubule barrier model), which was optimized using in silico computational modeling. The tri-culture MPS successfully filtered blood serum protein, resorbed glucose, and generated filtrate. This glomerulus and proximal convoluted tubule MPS is a novel system for both human-relevant testing and examining pharmacokinetic interactions.
\end{abstract}

Stephanie Y. Zhang and Gretchen J. Mahler*1

${ }^{1}$ Department of Biomedical Engineering, The State University of New York at Binghamton, NY, USA

* Correspondence: Corresponding Author gmahler@binghamton.edu

Keywords: kidney, cytoskeleton, microfluidics, cytotoxicity, pharmacokinetics

Abstract

Kidney microphysiological systems (MPS) serve as valuable preclinical instruments in recapitulating physiological conditions and determining underlying interactions involved in renal clearance and osmoregulation. Current kidney MPS models target individual regions of the nephron, such as the glomerulus and proximal tubule, but fail to incorporate multiple filtration and absorption interfaces. In the current study, an in vitro MPS features key filtration and reabsorption properties of the human glomerulus and proximal tubule for seven days of operation. Three human-derived cell types, including the conditionally immortalized human podocytes (CIHP-1), human umbilical vein endothelial cells (HUVECs), and human proximal tubule cells (HK-2), were adapted to serum-free medium prior to being seeded into the three-component MPS (T-Junction splitter, glomerular housing unit, and parallel proximal tubule barrier model), which was optimized using in silico computational modeling. The tri-culture MPS successfully filtered blood serum protein, resorbed glucose, and generated filtrate. This glomerulus and proximal convoluted tubule MPS is a novel system for both human-relevant testing and examining pharmacokinetic interactions. 


\section{Introduction}

Biopharmaceutical research and development faces a major productivity crisis in the depreciating efforts to develop novel drugs (Woodcock \& Woosley, 2008). Despite over 30 years of investment in biomedical sciences and the scientific tools used in drug discovery, few results have been well-translated in the preclinical and clinical stages (Giffin, Robinson, \& Olson, 2009; Wehling, 2006). The reliance on conventional cell culture systems and animal models during preclinical testing hinders the establishment of human-related kidney predictive models (Awdishu \& Mehta, 2017; Wilmer et al., 2016). Microphysiological systems (MPS) accurately model human systems in a compact, efficient fluidic tool that can introduce controlled spatiotemporal micro-environments (Kim \& Wu, 2012), support accurate human responses, allow for real-time imaging, encourage cell differentiation, and pinpoint cell-cell interactions under a variety of physiological conditions (Phillips et al., 2020; C. Sakolish et al., 2018; C. M. Sakolish, Esch, Hickman, Shuler, \& Mahler, 2016; C. M. Sakolish, Philip, \& Mahler, 2019; Wu et al., 2020). Recent developments in stem cell research (Musah et al., 2017; Sciancalepore et al., 2014), regenerative medicine (Gomes, Rodrigues, Domingues, \& Reis, 2017), biomaterials (Homan et al., 2016; van Midwoud, Janse, Merema, Groothuis, \& Verpoorte, 2012), tissue engineering (Jang et al., 2013; Jansen et al., 2014; Ng, Zhuang, Lin, \& Teo, 2012; Tourovskaia, Fauver, Kramer, Simonson, \& Neumann, 2014), and microfluidics allow for integration into three-dimensional (3D) MPS.

In the kidney, millions of nephrons employ filtration, reabsorption, secretion, and excretion processes. Each functional unit collaborates to filter out wastes and xenobiotics, separate water, ions, and small molecules from the blood, and recycle compounds to the blood (Figure 1A-B ) (Marieb \& Hoehn, 2007). Within the nephron, glomerular filtration consists of passive movement of plasma from glomerulus capillaries to the Bowman's capsule that is freely permeable to water and small solutes $\left(\mathrm{Na}^{+}\right.$, urea, and glucose), but not permeable to blood, white blood cells, platelets, or large molecular weight ( $>67 \mathrm{kDa}$ ) serum proteins (albumin) (Koeppen \& Stanton, 2012). The glomerular filtrate exits the glomerulus to enter a selective barrier of highly coiled tubules. There, the proximal convoluted tubule (PCT) utilizes active and passive transport to reabsorb glucose, sodium chloride, and water from the glomerular filtrate (Marieb \& Hoehn, 2007; Zanetti, 2020). During reabsorption, highly-concentrated filtrate becomes the leading site for nephrotoxin accumulation, a precursor for acute kidney injury or chronic kidney disease. Nearly $90 \%$ of renal toxicity cases are derived from both the glomerulus and PCT of the kidney (Bonventre, Vaidya, Schmouder, Feig, \& Dieterle, 2010; Jang et al., 2013; C. M. Sakolish et al., 2016). Current MPS designs of the kidney have been well established for the PCT segment of the nephron, representing only a section of the renal absorption process (Jang et al., 2013; C. Sakolish et al., 2020; C. Sakolish et al., 2018; C. M. Sakolish et al., 2016; C. M. Sakolish et al., 2019; Weber et al., 2016). However, incorporating both filtration and absorption interfaces will refine the physiological relevance of the kidney in vitro barrier model, and permit rapid screening for drug toxicity in preclinical studies.

In this study a novel, physiologically realistic MPS of the proximal tubule and glomerulus that incorporates cross flow filtration and is capable of long-term operation has been developed. The tri-culture system houses conditionally immortalized human podocytes (CIHP-1) to represent the ultrafiltration processes from the fenestrations in the glomerular capsule, human umbilical vein endothelial cells (HUVECs) for the capillaries recirculating solutes in the bloodstream, and human kidney-2 (HK-2) to recreate the reabsorption processes in the PCT. Key design requirements included integration of the glomerular filtration fraction (0.15-0.2) and tubular reabsorption (0.65-0.7) (Feher, 2017; Marieb \& Hoehn, 2007). The cells within the MPS were grown under dynamic flow engineered to mimic flow conditions in vivo (0.4-1.2 dyne-s $\left./ \mathrm{cm}^{2}\right)$ (Wilmer et al., 2016) for seven days, and then the system was challenged with fluorescein isothiocyanate- human serum albumin (FITC-HSA) to assess its filtration functional capacity. Cells within the device were imaged using confocal microscopy for attachment and cytoskeletal reorganization. MPS culture medium and imaging was completed without the introduction of animal by-products. This MPS introduces a novel definition of a PCT and glomerulus with physiological capability of blood serum protein filtration, glucose resorption, and filtrate formation capacity. 


\section{Materials and Methods}

\section{Simulating and Validating model in COMSOL MultiPhysics@}

A 2D computational model was developed through COMSOL Multiphysics (COMSOL Inc, Burlington, MA). Computational domains were generated using COMSOL's native geometry tools. To model the fluid flow in open and porous domains, the Free and Porous Media Flow physics package was used for porous membranes. Inlet, Outlet and No-Slip Wall conditions were used to define the boundary conditions of the computational domain. A symmetry boundary condition was used to reduce the computational load, and domains were discretized using a combination of swept, tetrahedral and boundary layer elements using native meshing tools. Using both stationary and time-dependent solvers, the 2D model was used to optimize tubing internal diameter (ID) and length (Cole-Parmer, Vernon Hills, IL), and total flow rate from the peristaltic pump (Watson-Marlow, Paramus, NJ). The primary design parameters were achieved a passive fluid flow split at the glomerular filter T-junction (Eldon James, Denver, CO), and obtaining a fluid shear stress of 0.65 dyn-s $/ \mathrm{cm}^{2}$ across the top chamber of the proximal tubule device (PCT), which mimics the physiological fluid shear stress in the human proximal tubule.

\section{Fabrication of the Microphysiological System}

Construction of the proximal tubule-on-a-chip has been previously described (C. M. Sakolish \& Mahler, 2017; C. M. Sakolish et al., 2019). Detailed description of the fabrication method is in the supplementary materials.

\section{Cell Culture}

All cell lines were adapted to Endothelial Serum Free Medium (ESFM, Thermo Fisher Scientific, Waltham, MA) supplemented with $10 \mu \mathrm{g} / \mathrm{mL}$ of human plasma fibronectin (Thermo Fisher Scientific, Waltham, MA), $20 \mathrm{ng} / \mathrm{mL}$ of human recombinant epidermal growth factor (EGF, Thermo Fisher Scientific, Waltham, $\mathrm{MA}$ ), and $10 \mathrm{ng} / \mathrm{mL}$ of human recombinant basic fibroblast growth factor (bFGF, Thermo Fisher Scientific, Waltham, MA). Conditionally immortalized human podocytes (CIHP-1) purchased from Dr. Saleem Moin's laboratory at the University of Bristol at passage 16 proliferated at $33^{\circ} \mathrm{C}$ in $5 \% \mathrm{CO}_{2}$ and were passaged every 10-14 days. The human, renal proximal tubule cell line (HK-2) (passage 20-30) obtained from the American Type Culture Collection (ATCC, Manassas, VA) was incubated at $37^{\circ} \mathrm{C}$ in $5 \% \mathrm{CO}_{2}$ and passaged every 5-7 days with 0.05\% Trypsin-EDTA (Thermo Fisher Scientific, Waltham, MA). Human umbilical vein endothelial cells (HUVECs) purchased from Lonza Bioscience (Rockland, MD) were maintained at $37^{\circ} \mathrm{C}$ in $5 \% \mathrm{CO}_{2}$ and experiments were completed using passages 3-7.

\section{CIHP-1, HUVECs, and HK-2 adhesion on porous membranes}

$30 \mathrm{~nm}$ pore size polyethersulfone (PES) membranes (Sterlitech, Kent, WA) were coated with glomerular extracellular matrix (GECM), which consist of tri-layer of $8 \mu \mathrm{g} / \mathrm{cm}^{2}$ rat-tail collagen 1 (BD Biosciences, Franklin Lakes, NJ), $8 \mu \mathrm{g} / \mathrm{cm}^{2}$ heparin sulfate (Sigma-Aldrich H7640), and $8 \mu \mathrm{g} / \mathrm{cm}^{2}$ collagen 1 (BD Biosciences, Franklin Lakes, NJ). The selection of the GECM composition was adapted from previous work (C. M. Sakolish \& Mahler, 2017; C. M. Sakolish et al., 2019). Detailed description of the adhesion methods is in the supplementary materials.

\section{Assembly of housing unit and microfluidic device}

The experimental study was conducted in a sterile, biosafety cabinet cleaned with $70 \%$ ethanol. All device components were autoclaved prior to experimentation. The internal and external components of the peristaltic pump were cleaned with $70 \%$ ethanol before stringing the Pharmed BPT $0.25 \mathrm{~mm} \mathrm{ID} \mathrm{tubing}$ 
(Cole-Parmer, Vernon Hills, IL) into the cassette with a conical tube of $1 \mathrm{X}$ phosphate buffered saline (PBS). All tubing was subjected to an average flow of $40 \mu \mathrm{L} / \mathrm{min}$ with $1 \mathrm{X}$ PBS for 1 hour to eradicate bubble formation. Assembly of the glomerulus stainless steel housing unit (Advantec MFS, Dublin, CA) (C. M. Sakolish et al., 2016; C. M. Sakolish \& Mahler, 2017; C. M. Sakolish et al., 2019), consisting of a stainless steel mesh (Advantec MFS, Dublin, CA), pre-cut $14 \mathrm{~mm}$ in diameter PES membrane (Sterlitech, Kent, WA), polytetrafluoroethylene (PTFE) gasket (Advantec MFS, Dublin, CA), pre-cut $200 \mu \mathrm{L}$ pipet tips (VWR International, Radnor, PA), and leur locks (Cole-Parmer, Vernon Hills, IL) was sealed with an autoclavable wrench (SteriTool, Jacksonville, FL). While the assembled glomerulus housing unit was attached to the end of the tubing to fill with $1 \mathrm{X}$ PBS, assembly of the T-junction and the CNC machined, polycarbonate PCT device was completed. Within the PCT device, a $0.4 \mu \mathrm{m}$ pore size polycarbonate membrane and 0.01-in thick silicone polymer gasket (McMaster-Carr, Elmhurst, IL) created anin vitro barrier system with an apical and basolateral sections. The components are assembled in their respective locations after filling with $1 \mathrm{X}$ PBS, and placeholder porous membranes were replaced with the cell seeded porous membranes. Afterwards, the inlet fluid was replaced with the supplemented ESFM incubated at $37^{\circ} \mathrm{C}$, and two outlet tubing was connected to $50 \mathrm{~mL}$ conical tube for filtrate output and bloodstream output. ESFM was changed every 2-3 days for a week.

\section{MPS Cellular Models}

Under dynamic conditions, two cellular models (single and tri-cultures) were employed with the glomerulus and PCT MPS components to assess the necessity of a multi-culture model. The single culture contained only proximal tubule cells (HK-2) adhered to the polycarbonate membrane in the PCT device. The single culture MPS was operated with the T-Junction, stainless-steel glomerulus housing unit with blank PES membrane, and seeded HK-2 cells in the PCT device. There was no glomerular or endothelial cells (CIHP-1 and HUVECs) on the PES membranes. The tri-culture housed all three cell-types on their respective membranes. The podocytes (CIHP-1) and endothelial (HUVECs) cells were attached to the PES membrane in the glomerulus unit in addition to the proximal tubule (HK-2) cells attached to the polycarbonate membrane in the PCT device.

\section{Volume Flow Assay}

Filtrate output volume was collected daily in $50 \mathrm{~mL}$ conical tubes (Corning, Corning, NY) and stored in $-20^{\circ} \mathrm{C}$ for future assessment. In post-experiment testing, the volumes were thawed, weighed, and normalized to an empty conical tube. Based on the density of the volume $(1 \mathrm{~g} / \mathrm{mL})$, total volume was calculated over time in $\mathrm{mL} / \mathrm{min}$.

\section{FITC-human serum albumin flow assay}

After seven days of fluidic culture, the nephron-on-a-chip system was subjected with $0.1 \mathrm{mg} / \mathrm{mL}$ of FITC-HSA (Abcam) diluted with 1X PBS by replacing the inlet conical tube fluid. In two black 96 well plates, samples $(\mathrm{n}=4)$ of the inlet and outlet fluid flow were collected at each 15-min intervals for $2 \mathrm{hrs}$. Fluorescence was assessed with Synergy Plate Reader (Biotek, Winooski, VT) and Gen5 software with an emission/excitation wavelength of $485 / 528 \mathrm{~nm}$. Results were normalized to the average maximum fluorescent intensity.

\section{Fluorescent staining and image processing}

Following the system challenge with HSA, membranes were removed from the devices and placed in a 4-well plate. The cells were fixed with $4 \%$ paraformaldehyde (PFA) diluted in $1 \mathrm{x}$ PBS for 5 min at room temperature and permeabilized with $0.1 \%$ Triton X-100 in 1x PBS for $15 \mathrm{~min}$. The cells were stained with phallodin 568 (Invitrogen) and counterstained with Hoescht 33342 (Thermo Fisher Scientific, Waltham, MA) for 30 mins. Membranes were then washed three times with 1X PBS for each 5 mins. Samples were mounted onto $24 \mathrm{x}$ 
40 x $0.13 \mathrm{~mm}$ glass slides through ProLong Gold and visualized with Zeiss Leica SP5 X confocal microscope. Images were processed in FIJI ImageJ (Schindelin et al., 2012) for directionality, confluence, and cell count. Z-stacks were taken for the membranes to generate a three-dimensional (3D) profile.

\section{Infinity glucose assay}

Glucose concentrations were calculated from the filtrate output volume using the Infinity Glucose Hexokinase Liquid Stable Reagent assay (Thermo Scientific, Waltham, MA). The reagent was diluted with filtrate output volume at a ratio of 150:1, incubated in $37 \mathrm{degC}$ for three minutes, and plated into a 96 well plate $(\mathrm{n}=4)$ for each day. Absorbance was assessed with Synergy Plate Reader (Biotek, Winooski, VT) and Gen5 software with a primary/secondary wavelength of $340 / 380 \mathrm{~nm}$. Standards were established with both a glucose solution of $1 \mathrm{mg} / \mathrm{mL}$ of $0.1 \%$ benzoic acid and supplemented ESFM.

\section{Statistical Analysis}

Nonparametric one-way ANOVA with post-hoc Dunn's test, Mann-Whitney test, and regression tests were conducted in GraphPad Prism(r) (GraphPad, San Diego, CA). All data from single- and tri-cultures were presented with medians and ranges.

\section{Results}

\section{A glomerular and proximal tubular microphysiological system}

Filtration and reabsorption of the glomerulus and PCT were assessed by two main devices in the construction of the MPS (Figure 1C-D ). Within the stainless steel housing unit (Figure 1C), the glomerulus enclosed a single porous PES membrane (porosity $=60$ ) seeded with the differentiated CIHP-1 and HUVECs. The differentiated podocytes were cultured onto the GECM and EECM coated PES membranes 14 days prior to initiation of flow. The negatively charged GECM and EECM generates a charge-selective filtration barrier of the glomerulus (C. M. Sakolish et al., 2016). During differentiation, podocytes were observed for flattened morphological characteristics with finger-like projections in areas surrounding PES membranes. On day 15, PES membranes were flipped and seeded with the endothelial cells for 4 hours. Once the HK-2 cells were seeded onto a fibronectin-coated polycarbonate membrane (porosity $=20$ ) for 4-hours, it was sealed in the machined bi-layer, polycarbonate microfluidic device described by previous work (Figure 1D ) (C. M. Sakolish \& Mahler, 2017; C. M. Sakolish et al., 2019). Its physical dimensions (30 mm long x $15 \mathrm{~mm}$ wide x $50 \mu \mathrm{m}$ thick) is comparatively similar to reported in vivo PCT dimensions in the human kidney (14 mm long x $40 \mu \mathrm{m}$ thick) (Lote \& Lote, 1994). The fully constructed MPS was connected to a T-Junction (Figure 2A-B ), a splitter of fluid flow to recycle $90 \%$ of flow into the bloodstream and $10 \%$ into the filtrate output. While the peristaltic pump maintained the MPS flow rate for the seven-day operation, additional modeling was required to optimize tubing length, diameter, and to safeguard physiological flow rates and shear rates (0.4 -1.5 dynes $\left./ \mathrm{cm}^{2}\right)$ within both glomerular housing unit and PCT microfluidic device.

\section{Flow characterization of microphysiological system}

To mediate the MPS microenvironment, a two-dimensional (2D) numerical simulation of porous media transport was performed using a finite element model of the device via COMSOL MultiPhysics@. A series of parametrizations (Table) was performed for the velocity flow $(\mathrm{m} / \mathrm{s})$ (Figure 3A-B ) and shear rate $(1 / \mathrm{S})$ (Figure 3C-D ) based on the assumptions of steady state, constant fluid flow, viscosity and density, incompressible flow, minimal fluid evaporation, and negligible gravity. Conversions into physiological measurements were adopted using the following shear stress (Equation 1 ).

$\tau=\frac{6 Q \mu}{b h^{2}}($ Equation 1$)$ 
where $\mu$ is the endothelial serum free culture medium viscosity at $37{ }^{\circ} \mathrm{C} ; \tau$ is the shear stress $\left(\right.$ dyn-s $\left./ \mathrm{cm}^{2}\right) ; Q$ is the volumetric flow rate $\left(\mathrm{cm}^{3} / \mathrm{s}\right) ; b$ is the channel width; and $h$ is the channel height $\left(Q=7.6 \times 10^{-4} \mathrm{~cm}^{3} / \mathrm{s}\right.$, $\mu=8.9 \times 10^{-3}$ dyn-s $\left./ \mathrm{cm}^{2}, h=0.05 \mathrm{~mm}, b=30 \mathrm{~mm}\right)$

Based on the conversions, the optimal tubing length of filtrate output and bloodstream were $\sim 508 \mathrm{~mm}$ and $734 \mathrm{~mm}$ respectively. The tubing length ranges were selected based on peristaltic pump and incubator sizing constraints. The inlet mass flow rate was $7.6 \times 10^{-7} \mathrm{~kg} / \mathrm{s}\left(7.6 \times 10^{-4} \mathrm{~cm}^{3} / \mathrm{s}\right)$ to generate a shear stress of 0.5 dyne-s $/ \mathrm{cm}^{2}$ across the apical layer of the PCT device. Means of 1-D velocity profiles from filtrate output and the bloodstream had a ratio of $83 / 81$ (Figure 3 ). Concentration profiles of $0.1 \mathrm{mg} / \mathrm{mL}$ FITC-HSA were computed using its diffusivity coefficient $\left(61 \mathrm{\mu m}^{2} / \mathrm{s}\right)$ in Transport by Diluted Species with a timedependent solver from 0-120 minutes (Figure 3E-F ). The concentration 1-D profile was characterized with an exponential increase until it plateaued and approached steady state around 105-120 minutes. While these simulations are effective in determining the optimal geometry for the desired shear stress, the model was idealistic and reproduced without the inclusion of cellular effects that may alter the overall outcome.

Inlet mass flow rate $(\mathrm{kg} / \mathrm{s})$ was experimentally validated by using a single inlet $0.25 \mathrm{ID} \mathrm{mm}$ tubing at $4-16$ rpm pump rate $\left(0.19-0.72\right.$ dyne-s $\left./ \mathrm{cm}^{2}\right)$ (Figure 4A-B $)$. Flow rates $(\mu \mathrm{L} / \mathrm{min})$ and shear stress $\left(\right.$ dyne-s $\left./ \mathrm{cm}^{2}\right)$ was determined with pumping $1 \mathrm{X}$ phosphate buffered saline (PBS) over the course of 2 hours, and collecting samples at 15 -minute intervals $(\mathrm{n}=4$ for each $\mathrm{rpm})$. At $12 \mathrm{rpm}$ pump rate, the flow inlet $\left(\mathrm{Q}_{\text {in }}\right)$ achieved an average of $45.3 \pm 3.0 \mu \mathrm{L} / \mathrm{min}$ with a shear rate of $0.5 \pm 0.04$ dyne-s $/ \mathrm{cm}^{2}$. With the assembled MPS, $1 \mathrm{X}$ PBS was cyclically pumped for the same duration, and filtrate formation $\left(Q_{\text {primary filtrate }}\right)$ and bloodstream recycling ( $Q_{\text {blood }}$ ) rates were an average of $16.2 \pm 4.5 \mu \mathrm{L} / \mathrm{min}$ and $29.0 \pm 4.5 \mu \mathrm{L} / \mathrm{min}$, respectively. Filtrate output and shear stress across the PCT for the single culture and tri-culture conditions (Figure 4C-D ) were compared with the simulated and experimental settings to characterize the system under non-ideal conditions. Single culture devices contained only the proximal tubule (HK-2) cells seeded into the PCT device, while the tri-culture was seeded with CIHP-1 and HUVECs in the glomerulus housing unit along with the HK-2 cells in the PCT. Implementation of single culture is representative of pre-established PCT devices (Homan et al., 2016; Jang et al., 2013; Jansen et al., 2016; Ng et al., 2012; Raghavan, Rbaibi, Pastor-Soler, Carattino, \& Weisz, 2014; Sabbisetti et al., 2014; C. Sakolish et al., 2020; C. Sakolish et al., 2018; Weber et al., 2016), whereas tri-cultures reflect the combination of the human glomerulus and PCT. While the $90.0 \%$ of fluid was not recycled into the bloodstream as shown in the simulation, approximately $36.0 \%$ was outputted into the waste collection and $64.0 \%$ was recycled into the MPS.

\section{Recapitulation of glomerulus and proximal tubule functions}

Key characteristics of a functional nephron are its blood serum protein filtration, glucose resorption, and filtrate formation capacity. Two culture conditions were established to demonstrate system characteristic differences, which was denoted as a single $(n=3)$ and tri-culture (all cell-types) $(n=3)$. Throughout the seven-day operation, primary filtrate formation was collected every 24 hours in conical tubes and immediately stored at $-20^{\circ} \mathrm{C}$ before additional assessment. Volumes of waste for each culture type were weighed to measure velocity flow rate $(\mathrm{mL} / \mathrm{min}$ ) (Figure 5A-B ). The primary filtrate formation in the single-culture maintained a median of approximately $30 \mu \mathrm{L} / \mathrm{min}$, while the tri-culture had $15.0 \mu \mathrm{L} / \mathrm{min}$. The introduction of the glomerular cells and endothelial cells created a barrier effect, reducing the flow rate within the apical PCT of the MPS to generate a shear stress from 0.3 to 0.2 dyne-s $/ \mathrm{cm}^{2}$. Results of the simulated, experimental, single, and tri-culture filtrate output and shear stress across the apical region of the PCT were shown (Figure 4CD ). While the simulated model exhibited a linear behavior based on its regression $\left(R^{2}=1.0\right)$, the shear stress under experimental conditions significantly decreased in the presence of cells, suggesting that simulations provided only ideal conditions and required further parametrizations.

Since the proximal tubule is responsible for nearly $90.0 \%$ of glucose reabsorption in the kidney, glucose secretion $(\mathrm{mg} / \mathrm{mL})$ in both single and tri-culture devices were evaluated using the Infinity Glucose Assay (Figure 5C-D ). Filtrate output volumes were sampled three times into a 96 well plate and compared to a $1 \mathrm{mg} / \mathrm{mL}$ glucose solution diluted with $0.1 \%$ benzoic acid standard curve. Samples were read with the 
plate reader at an absorbance of $340 \mathrm{~nm}$. Both culture conditions lacked statistically significant difference due to the large volume of daily filtrate output $(\sim 30-40 \mathrm{~mL} /$ day $)$, although there was a downward trend in the amount of glucose present in the filtrate output over the course of the 7 day culture, indicating some proximal tubule function. No significant change in both velocity and glucose concentrations (Figure 5B and $\mathbf{D}$ ) demonstrated that the MPS was stable during the seven days.

On Day 7 of MPS operation, $0.1 \mathrm{mg} / \mathrm{mL}$ of fluorescently tagged human serum albumin (FITC-HSA, Abcam \#ab8030) diluted with 1X PBS was circulated into the MPS to assess glomerulus function and were analyzed for the amount of unfiltered serum proteins in both the filtrate outlet and blood stream circuit reservoir (McQuarrie, Shakerdi, Jardine, Fox, \& Mackinnon, 2011). Samples were drawn from the filtrate outlet and blood stream circuit reservoirs every 15 minutes for $2 \mathrm{hrs}$ and placed in a black, 96-well plate. Samples were analyzed for fluorescence with a Synergy 2 plate reader against a standard curve (Figure 5E-G ). Protein concentration in the bloodstream minimally declined from $0.1 \mathrm{mg} / \mathrm{mL}$ of FITC-HSA for both case conditions. In the tri-culture conditions, there was a statistically significant difference $(\mathrm{p}<0.001)$ between filtrate output and bloodstream circuit output (Figure 5F ). FITC-HSA concentration in tri-culture in the blood circuit output $(0.083 \mathrm{mg} / \mathrm{mL})$ was 8 times greater than the filtrate output $(0.011 \mathrm{mg} / \mathrm{mL})$, suggesting that there is a functional filtration process in the tri-culture MPS. This behavior was not exhibited in the single culture conditions. Single-cell culture exhibited an increase in protein concentrations in the filtrate output, while the tri-culture devices had little protein reach the filtrate output (Figure 5G ). Absence of protein in the waste or filtrate stream suggests that the glomerular cells prevent the FITC-HSA from passing through the glomerular membrane, similar to the function of the glomerulus in vivo .

Fluorescent stains provided insight into the cellular phenotype following dynamic culture. The membranes, PCT (polycarbonate) and glomerular (PES) membranes from the MPS were removed, fixed with $4 \%$ paraformaldehyde (PFA), stained with phallodin 568) and Hoescht 33342, and imaged using a Zeiss confocal microscope at 40x magnification. F-actin cytoskeleton restructuring was imaged with phallodin 568, whereas DNA in the nuclei was counterstained with Hoescht 33342. To determine the cellular confluence, qualitative $2 \mathrm{D}$ orthogonal views and three-dimensional (3D) views (Figure 6A-F ) were obtained. Qualitatively, the HK-2 cells on the PCT membrane (Figure 6A-B ) demonstrated a monolayer behavior as previously shown (C. M. Sakolish \& Mahler, 2017), and quantitatively represented by the $75.3 \%$ confluence and significantly greater cell count in comparison to podocytes and endothelial cells (Figure 6G-H ). The CIHP-1 cells on the glomerular membrane appeared flatter, fewer in cell count, and fewer F-actin across the plane due to a larger average cell area $\left(831.1 \mu \mathrm{m}^{2}\right)$ compared to HK-2 $\left(183.6 \mu \mathrm{m}^{2}\right)$ and HUVECs $\left(353.7 \mu \mathrm{m}^{2}\right)$. From the $3 \mathrm{D}$ view of Figure $\mathbf{6 C}$, finger like projections also validated full differentiation (day 14). The endothelial cells were highly directional in the presence of flow, but attachment was limited by day 7 (Figure 6E-F ). This may be related to HUVECs poor ability to adapt to the dynamic crossflow culture conditions.

\section{Discussion}

Previous renal MPS models have targeted specific structures of the nephron, but regulation of xenobiotics and substrates in the blood is a concerted action of both filtration in the glomeruli and active reabsorption by the proximal tubule. The challenge in establishing an effective nephron MPS originates from fulfilling all required characteristics of kidney MPS: incorporation of renal cellular models (tubular structures or in vitro barrier models) (Jang et al., 2013; C. Sakolish et al., 2020; C. Sakolish et al., 2018; C. M. Sakolish et al., 2016; Tourovskaia et al., 2014; Weber et al., 2016), functional (filtration, absorption, and secretion) processes, and drug toxicity/ recovery testing. In this study, the glomerulus- and PCT- MPS addresses most of the features of a renal MPS, while also maintaining in vivo shear stress across the apical PCT (0.4-1.5 dyne-s $/ \mathrm{cm}^{2}$ ) and recirculating $64.0 \%$ of flow into the bloodstream and $36.0 \%$ into the filtrate output. The tri-culture system of CIHP-1, HUVECs and HK-2 cells provides a novel, realistic approach by recapitulating the critical functions in both the glomerulus and PCT for long-term, overcoming the limitations of existing glomerulus-on-a-chip and PCT-on-a-chip designs, and assessing for drug toxicity. 
Geometrically, many vital attributes contribute to the innovation of the glomerulus and PCT- MPS that have never been modeled previously in vitro. The MPS presents a dual in vitro barrier model in the glomerular filtration chamber and the PCT reabsorption device. Within the glomerular unit, the 30nm pore size PES membrane was selected based on the physiological size-exclusive filtration found on the human glomerular capillary walls (C. M. Sakolish \& Mahler, 2017; C. M. Sakolish et al., 2019). The PCT barrier model highlights a multi-layered fluidic platform separated by a porous membrane, creating a dual channel system (apical and basolateral). Proximal tubule cells on the membrane selectively resorb solutes from the apical filtrate formation line to the basolateral bloodstream, reenacting the renal active and passive transport processes. This recirculation of flow was established through the T-Junction component, crucial in bloodstream circuit reservoir to simulate the removal of compounds that have been reabsorbed by the renal tubule. Additionally, the PCT device was constructed in a polycarbonate material instead of the traditional polydimethylsiloxane (PDMS), meaning that the devices were highly robust, autoclavable, and reused up to 10 times. Failure points, such as leakage, occurred in the presence of long-term exposure to humidity, high temperatures, and shear stress. Yet, the MPS design fosters low-cost manufacturing and minimizes wasted resources in single-use devices.

Throughout the MPS experiments, comparisons of the single and tri-culture systems determined the significance of modeling concerted structures of the nephron. The single culture is illustrative of the conventional PCT devices (Jang et al., 2013; Ng et al., 2012; Raghavan et al., 2014; C. M. Sakolish et al., 2019; Weber et al., 2016), but the unique attributes of the glomerulus and MPS system include three cell types, selected based on its key functions in filtration, reabsorption, and secretion. Filtration is operated through the glomerular filtration barrier mainly controlled by the glomerular and endothelial cells (Li et al., 2016). Co-culturing these cells have previously proved challenging through the technical difficulties of glomerular endothelial cells and podocytes. Since both cell types are highly differentiated, specialized, and interdependent cell types (Xinaris et al., 2016; Xinaris et al., 2012), selection of the cells in the model is vital in evaluating the MPS. CIHP-1 cells, representative of the podocytes, were selected based on its immortalized, homogenous characteristics. To ensure that the MPS establishes efficacy, homogeneity minimizes outliers and inconsistencies. Similarly, HUVECs were selected based on prevalent use in endothelial cell studies (Caulfield \& Farquhar, 1974; Venturoli \& Rippe, 2005) and derivation from humans. Reabsorption is mostly contributed by the proximal tubule cells as denoted by the HK-2 cells, which were selected based on extensive use previous work (Jang et al., 2013; C. M. Sakolish et al., 2016; C. M. Sakolish \& Mahler, 2017; C. M. Sakolish et al., 2019). From the comparative results, the single culture and tri-culture were indicative of the significance of establishing a multi-cellular model in future kidney research. The differences in velocity flow rate and protein concentrations validate how the glomerulus and PCT MPS greatly impact the retention of functional cell-cell and cell-tissue interactions.

To achieve animal-byproduct free culture conditions, multiple media types were tested. Based on the results of the viability and proliferation testing (Figure S1), all three cell types were adapted to an optimal common cell culture medium (ESFM) prior to dynamic experimentation. For the glomerular functional assay, human serum albumin was used (FITC-HSA, 67.0 kDa). Cells within the glomerulus and PCTMPS demonstrated an in vivo - like functional activity through the retention of HSA $(\sim 67 \mathrm{kDa})$ from circulating in the bloodstream pathway, while resorbing concentrations of glucose in filtrate output. The protein concentrations were depicted in both the plate reader fluorescence readings, but also in the confocal images, where there was an uptake of the green fluorescent protein in the podocytes (Figure S2 ). Previous works (Jang et al., 2013; Ng et al., 2012) found that dynamic conditions results in cytoskeletal reorganization and junctional reformation in PCT-derived cells. These outcomes were not clearly observed in the glomerulus and PCT MPS due to the low shear stress to exhibit healthy renal conditions and longer culture time. While human serum albumin results in this study do not distinguish between glomerular filtration and tubular reabsorption, a previous glomerulus and PCT MPS iteration (C. M. Sakolish \& Mahler, 2017) featured transport with other proteins sizes. The MPS with only endothelial (HUVECs) and proximal tubule (HK-2) cells was subjected with $0.1 \mathrm{mg} / \mathrm{mL}$ of FITC-bovine serum albumin (FITC-BSA, $66.4 \mathrm{kDa}$ ) and FITCovalbumin $(45.0 \mathrm{kDa})$. The HUVECs demonstrated more BSA localization in the junctions of the cell 
monolayer, whereas the HK-2 had an increase in BSA in vesicles, suggesting a presence of passive and active transport, respectively. Future works can incorporate different sized fluorescent compounds (i.e. dextran) to characterize the specificity of trans-glomerular filtration and tubular reabsorption (Caulfield \& Farquhar, 1974; Ng et al., 2012; Venturoli \& Rippe, 2005; Xinaris et al., 2016; Xinaris et al., 2012). For imaging in post-dynamic experiments, immunocytochemistry would typically be used to display functional biomarkers, such as VE-cadherin, clathrin, or nephrin (Musah et al., 2017; Petrosyan et al., 2019; Weber et al., 2016). In this study, alternate stains were used to avoid the use of animal products (Calcein AM, Hoescht 3342, and phallodin 568). Largely, the glomerulus- and PCT- MPS offers an effective tool for assessing preclinical

drug adsorption, distribution, metabolism, elimination, and toxicity (ADMET) testing, modeling disease for drug treatment regime trials, and understanding cellular crosstalk.

Additional work is required to ensure confluent endothelial cell adhesion by day 7 on the PES membrane. Previous works have identified Collagen IV and laminin as a more physiological relevant extracellular matrix in renal studies (D. R. Abrahamson, 1991; D. R. Abrahamson, Hudson, Stroganova, Borza, \& John, 2009; John \& Abrahamson, 2001; Li et al., 2016; Mauer, 1994; Pavenstadt, Kriz, \& Kretzler, 2003; C. Sakolish et al., 2018; Weber et al., 2016). Initially, glomerular endothelial and epithelial cells generate a glomerular basement membrane (GBM), a cytoskeletal template for renal cell adhesion. Following, fully differentiated podocytes further assemble matrix molecules into a highly intricate meshwork of collagen IV, laminin, heparin sulfate, entactin, agrin, and perlecan (D. Abrahamson, 1987; D. R. Abrahamson, 1985; Adler, 1992; John \& Abrahamson, 2001; Miner, 1998; Sariola, 1984). Of those extracellular proteins, type IV collagen and laminin have ensured endothelial monolayers and better preservation of the podocyte phenotype based on the F-actin distribution, nephrin, and synaptopodin (Li et al., 2016). Recreating a kidney diseased model, such as chronic kidney disease and drug-induced kidney injuries (DIKI), on a molecular scale is essential in innovating safer therapeutic agents. Establishing predictive models of DIKI avoid unnecessary risk for patients and reduce financial burden of compound attrition in pharmaceutical development. Further investigation of renal drug candidates, such as cisplatin, gentamicin, perfluorooctanoic acid (PFOA), and angiotensin-converting enzyme inhibitors (ACEI) would permit a wider scope into the pharmacokinetics within the glomerular and proximal tubule MPS. In Sakolish et. al, 2019, a similar glomerulus and PCT MPS with only HK-2 cells was implemented to test altered glomerular filtration rate (GFR), hyperglycemia, nephrolithiasis, and drug-induced nephrotoxicity. Four conditions were subjected in the MPS with their respective disease model: (1) physiological flow of 0.8 dyne-s $/ \mathrm{cm}^{2}$ and high shear flow of 5 dyne-s $/ \mathrm{cm}^{2}$, (2) KSFM and glucose-treated SFM, (3) $0.3 \mathrm{mg} / \mathrm{ml}$ of calcium oxalate monohydrate crystals (COM), and (4) $1.4 \mathrm{mM}$ of cisplatin and $0.27 \mathrm{mM}$ of cyclosporine exposures for $72 \mathrm{hrs}$ at $24 \mathrm{hr}$ intervals. Based on the significant changes in cellular responses of these single culture MPS compared to the static conditions (Figure S3 ), this operates as a precursor for the current glomerulus and PCT tri-culture MPS's potential application in assessing DIKI. More functional assays would build confidence in the physiologically credible characteristics. MPS under fluid-driven flow would have increased mechanical signaling in the primary cilia, microvilli, or glycocalyx, increased trafficking and expression of apical and basolateral transporters, and increased epithelial barrier function (Koepsell, 2013; Nieskens et al., 2016; Nieskens \& Wilmer, 2016; Phillips et al., 2020).

\section{Conclusion}

The glomerulus and proximal tubule microphysiological system presented in this study successfully recapitulates kidney filtration and reabsorption properties. A 2D computational model of the velocity and shear rate profiles provided a template for achieving a shear stress of $0.4-1.5$ dynes $/ \mathrm{cm}^{2}$ within the device. Single and tri-cellular in vitro models were characteristically different in their functional capabilities. Though both systems operate at 0.7 dynes $/ \mathrm{cm}^{2}$ for 7 -days, there are clear differences in the filtrate output. The significance of the tri-culture is verified by the glomerular cells filtering human serum albumin, acting as filtration barrier both biologically and mechanically. In this work, the tri-culture presents a more realistic cellular model of the glomerulus and PCT. 


\section{Conflicts of Interest}

The authors declare that the research was conducted in the absence of any commercial or financial relationships that could be construed as a potential conflict of interest.

\section{Funding}

This work was funded by the Alternative Research and Development Foundation.

\section{Acknowledgments}

We would like to thank Dr. Mei-Hsui Chen and Binghamton University Statistical Consulting Center for their assistance on all statistical analysis and discussions. We would also like to thank Joshua France for assistance with cellular growth curves.

\section{Reference}

Abrahamson, D. (1987). Structure and development of the glomerular capillary wall and basement membrane.American Journal of Physiology-Renal Physiology, 253 (5), F783-F794.

Abrahamson, D. R. (1985). Origin of the glomerular basement membrane visualized after in vivo labeling of laminin in newborn rat kidneys. The Journal of cell biology, 100 (6), 1988-2000.

Abrahamson, D. R. (1991).Glomerulogenesis in the developing kidney. Paper presented at the Seminars in nephrology.

Abrahamson, D. R., Hudson, B. G., Stroganova, L., Borza, D.-B., \& John, P. L. S. (2009). Cellular origins of type IV collagen networks in developing glomeruli. Journal of the American Society of Nephrology, 20 (7), 1471-1479.

Adler, S. (1992). Characterization of glomerular epithelial cell matrix receptors. The American journal of pathology, 141 (3), 571.

Awdishu, L., \& Mehta, R. L. (2017). The 6R's of drug induced nephrotoxicity. BMC nephrology, 18 (1), 124.

Bonventre, J. V., Vaidya, V. S., Schmouder, R., Feig, P., \& Dieterle, F. (2010). Next-generation biomarkers for detecting kidney toxicity. Nature biotechnology, 28 (5), 436-440.

Caulfield, J. P., \& Farquhar, M. G. (1974). The permeability of glomerular capillaries to graded dextrans: Identification of the basement membrane as the primary filtration barrier. The Journal of cell biology, 63 (3), 883-903.

Feher, J. J. (2017).Quantitative human physiology: an introduction: Academic press.

Giffin, R., Robinson, S., \& Olson, S. (2009). Accelerating the development of biomarkers for drug safety: workshop summary: National Academies Press.

Gomes, M. E., Rodrigues, M. T., Domingues, R. M., \& Reis, R. L. (2017). Tissue engineering and regenerative medicine: new trends and directions - a year in review. Tissue Engineering Part B: Reviews, 23 (3), 211-224. 
Homan, K. A., Kolesky, D. B., Skylar-Scott, M. A., Herrmann, J., Obuobi, H., Moisan, A., \& Lewis, J. A. (2016). Bioprinting of 3D convoluted renal proximal tubules on perfusable chips. Scientific reports, 6 , 34845 .

Jang, K.-J., Mehr, A. P., Hamilton, G. A., McPartlin, L. A., Chung, S., Suh, K.-Y., \& Ingber, D. E. (2013). Human kidney proximal tubule-on-a-chip for drug transport and nephrotoxicity assessment. Integrative Biology, 5 (9), 1119-1129.

Jansen, J., Fedecostante, M., Wilmer, M., Peters, J., Kreuser, U., Van Den Broek, P., . . . Wetzels, J. (2016). Bioengineered kidney tubules efficiently excrete uremic toxins.Scientific reports, 6 , 26715.

Jansen, J., Fedecostante, M., Wilmer, M., Van den Heuvel, L., Hoenderop, J., \& Masereeuw, R. (2014). Biotechnological challenges of bioartificial kidney engineering.Biotechnology advances, 32 (7), 1317-1327.

John, P. L. S., \& Abrahamson, D. R. (2001). Glomerular endothelial cells and podocytes jointly synthesize laminin-1 and-11 chains. Kidney international, 60 (3), 1037-1046.

Kim, B. J., \& Wu, M. (2012). Microfluidics for mammalian cell chemotaxis. Annals of biomedical engineering, $40(6), 1316-1327$.

Koeppen, B. M., \& Stanton, B. A. (2012). Renal Physiology E-Book: Mosby Physiology Monograph Series : Elsevier Health Sciences.

Koepsell, H. (2013). The SLC22 family with transporters of organic cations, anions and zwitterions. Molecular aspects of medicine, 34 (2-3), 413-435.

Li, M., Corbelli, A., Watanabe, S., Armelloni, S., Ikehata, M., Parazzi, V., . . . Lazzari, L. (2016). Threedimensional podocyte - endothelial cell co-cultures: assembly, validation, and application to drug testing and intercellular signaling studies. European Journal of Pharmaceutical Sciences, 86 , 1-12.

Lote, C. J., \& Lote, C. J. (1994).Principles of renal physiology : Springer.

Marieb, E. N., \& Hoehn, K. (2007).Human anatomy \& physiology : Pearson education.

Mauer, S. M. (1994). Structural-functional correlations of diabetic nephropathy. Kidney international, 45 (2), 612-622.

McQuarrie, E. P., Shakerdi, L., Jardine, A. G., Fox, J. G., \& Mackinnon, B. (2011). Fractional excretions of albumin and $\operatorname{IgG}$ are the best predictors of progression in primary glomerulonephritis. Nephrology Dialysis Transplantation, 26 (5), 1563-1569.

Miner, J. H. (1998). Developmental biology of glomerular basement membrane components. Current opinion in nephrology and hypertension, 7 (1), 13-19.

Musah, S., Mammoto, A., Ferrante, T. C., Jeanty, S. S., Hirano-Kobayashi, M., Mammoto, T., . . . Ingram, M. (2017). Mature induced-pluripotent-stem-cell-derived human podocytes reconstitute kidney glomerularcapillary-wall function on a chip.Nature biomedical engineering, 1 (5), 1-12.

Ng, C. P., Zhuang, Y., Lin, A. W. H., \& Teo, J. C. M. (2012). A fibrin-based tissue-engineered renal proximal tubule for bioartificial kidney devices: development, characterization and in vitro transport study. International Journal of Tissue Engineering, 2013 .

Nieskens, T. T., Peters, J. G., Schreurs, M. J., Smits, N., Woestenenk, R., Jansen, K., . . . Wilmer, M. J. (2016). A human renal proximal tubule cell line with stable organic anion transporter 1 and 3 expression predictive for antiviral-induced toxicity. The AAPS journal, 18 (2), 465-475.

Nieskens, T. T., \& Wilmer, M. J. (2016). Kidney-on-a-chip technology for renal proximal tubule tissue reconstruction. European journal of pharmacology, 790 , 46-56. 
Pavenstadt, H., Kriz, W., \& Kretzler, M. (2003). Cell biology of the glomerular podocyte.Physiological reviews, 83 (1), 253-307.

Petrosyan, A., Cravedi, P., Villani, V., Angeletti, A., Manrique, J., Renieri, A., . . . Da Sacco, S. (2019). A glomerulus-on-a-chip to recapitulate the human glomerular filtration barrier. Nature communications, 10 (1), 1-17.

Phillips, J. A., Grandhi, T. S. P., Davis, M., Gautier, J.-C., Hariparsad, N., Keller, D., . . . Van Vleet, T. R. (2020). A pharmaceutical industry perspective on microphysiological kidney systems for evaluation of safety for new therapies. Lab on a Chip, 20 (3), 468-476.

Raghavan, V., Rbaibi, Y., Pastor-Soler, N. M., Carattino, M. D., \& Weisz, O. A. (2014). Shear stressdependent regulation of apical endocytosis in renal proximal tubule cells mediated by primary cilia. Proceedings of the National Academy of Sciences, 111 (23), 8506-8511.

Sabbisetti, V. S., Waikar, S. S., Antoine, D. J., Smiles, A., Wang, C., Ravisankar, A., . . . Lee, M. (2014). Blood kidney injury molecule-1 is a biomarker of acute and chronic kidney injury and predicts progression to ESRD in type I diabetes. Journal of the American Society of Nephrology, 25 (10), 2177-2186.

Sakolish, C., Chen, Z., Dalaijamts, C., Mitra, K., Liu, Y., Fulton, T., . . . Chiu, W. A. (2020). Predicting tubular reabsorption with a human kidney proximal tubule tissue-on-a-chip and physiologically-based modeling. Toxicology in Vitro, 63, 104752.

Sakolish, C., Weber, E. J., Kelly, E. J., Himmelfarb, J., Mouneimne, R., Grimm, F. A., . . . Chiu, W. A. (2018). Technology transfer of the microphysiological systems: a case study of the human proximal tubule tissue chip. Scientific reports, 8 (1), 1-14.

Sakolish, C. M., Esch, M. B., Hickman, J. J., Shuler, M. L., \& Mahler, G. J. (2016). Modeling barrier tissues in vitro: methods, achievements, and challenges.EBioMedicine, 5, 30-39.

Sakolish, C. M., \& Mahler, G. J. (2017). A novel microfluidic device to model the human proximal tubule and glomerulus. RSC advances, 7 (8), 4216-4225.

Sakolish, C. M., Philip, B., \& Mahler, G. J. (2019). A human proximal tubule-on-a-chip to study renal disease and toxicity. Biomicrofluidics, 13 (1), 014107.

Sariola, H. (1984). Incomplete fusion of the epithelial and endothelial basement membranes in interspecies hybrid glomeruli. Cell differentiation, 14 (3), 189-195.

Schindelin, J., Arganda-Carreras, I., Frise, E., Kaynig, V., Longair, M., Pietzsch, T., . . . Schmid, B. (2012). Fiji: an open-source platform for biological-image analysis.Nature methods, 9 (7), 676-682.

Sciancalepore, A. G., Sallustio, F., Girardo, S., Passione, L. G., Camposeo, A., Mele, E., . . . Pisignano, D. (2014). A bioartificial renal tubule device embedding human renal stem/progenitor cells. PloS one, 9 (1), e87496.

Tourovskaia, A., Fauver, M., Kramer, G., Simonson, S., \& Neumann, T. (2014). Tissue-engineered microenvironment systems for modeling human vasculature. Experimental biology and medicine, 239 (9), 1264-1271.

van Midwoud, P. M., Janse, A., Merema, M. T., Groothuis, G. M., \& Verpoorte, E. (2012). Comparison of biocompatibility and adsorption properties of different plastics for advanced microfluidic cell and tissue culture models. Analytical chemistry, 84 (9), 3938-3944.

Venturoli, D., \& Rippe, B. (2005). Ficoll and dextran vs. globular proteins as probes for testing glomerular permselectivity: effects of molecular size, shape, charge, and deformability. American Journal of PhysiologyRenal Physiology, 288 (4), F605-F613.

Weber, E. J., Chapron, A., Chapron, B. D., Voellinger, J. L., Lidberg, K. A., Yeung, C. K., . . . Neumann, T. (2016). Development of a microphysiological model of human kidney proximal tubule function. Kidney 
international, 90 (3), 627-637.

Wehling, M. (2006). Translational science in medicine. International journal of pharmaceutical medicine, $20(5), 303-310$.

Wilmer, M. J., Ng, C. P., Lanz, H. L., Vulto, P., Suter-Dick, L., \& Masereeuw, R. (2016). Kidney-on-a-chip technology for drug-induced nephrotoxicity screening. Trends in biotechnology, 34 (2), 156-170.

Woodcock, J., \& Woosley, R. (2008). The FDA critical path initiative and its influence on new drug development. Annu. Rev. Med., 59 , 1-12.

Wu, Q., Liu, J., Wang, X., Feng, L., Wu, J., Zhu, X., . . . Gong, X. (2020). Organ-on-a-chip: recent breakthroughs and future prospects. BioMedical Engineering OnLine, 19 (1), 9.

Xinaris, C., Benedetti, V., Novelli, R., Abbate, M., Rizzo, P., Conti, S., . . . Cavallotti, D. (2016). Functional human podocytes generated in organoids from amniotic fluid stem cells. Journal of the American Society of Nephrology, 27 (5), 1400-1411.

Xinaris, C., Benedetti, V., Rizzo, P., Abbate, M., Corna, D., Azzollini, N., . . . Morigi, M. (2012). In vivo maturation of functional renal organoids formed from embryonic cell suspensions. Journal of the American Society of Nephrology, 23 (11), 1857-1868.

Zanetti, F. (2020). Kidney-on-a-chip Organ-on-a-chip (pp. 233-253): Elsevier.

\begin{tabular}{llll}
\hline Inlet Mass Flow Rate $\left(\mathbf{1 0}^{-\mathbf{7}} \mathbf{~} \mathbf{~ g} / \mathbf{s}\right)$ & Tubing ID Diameter $\mathbf{( m m})$ & Filtrate Output Length $\mathbf{( m m})$ & Bloodstrea \\
\hline 2.66 & 0.25 & 457 & 622 \\
5.15 & & 474 & 660 \\
6.67 & & 491 & 698 \\
7.55 & 0.51 & 508 & 736 \\
10.1 & & 525 & 774 \\
\hline
\end{tabular}

Table. MPS Parameterizations.

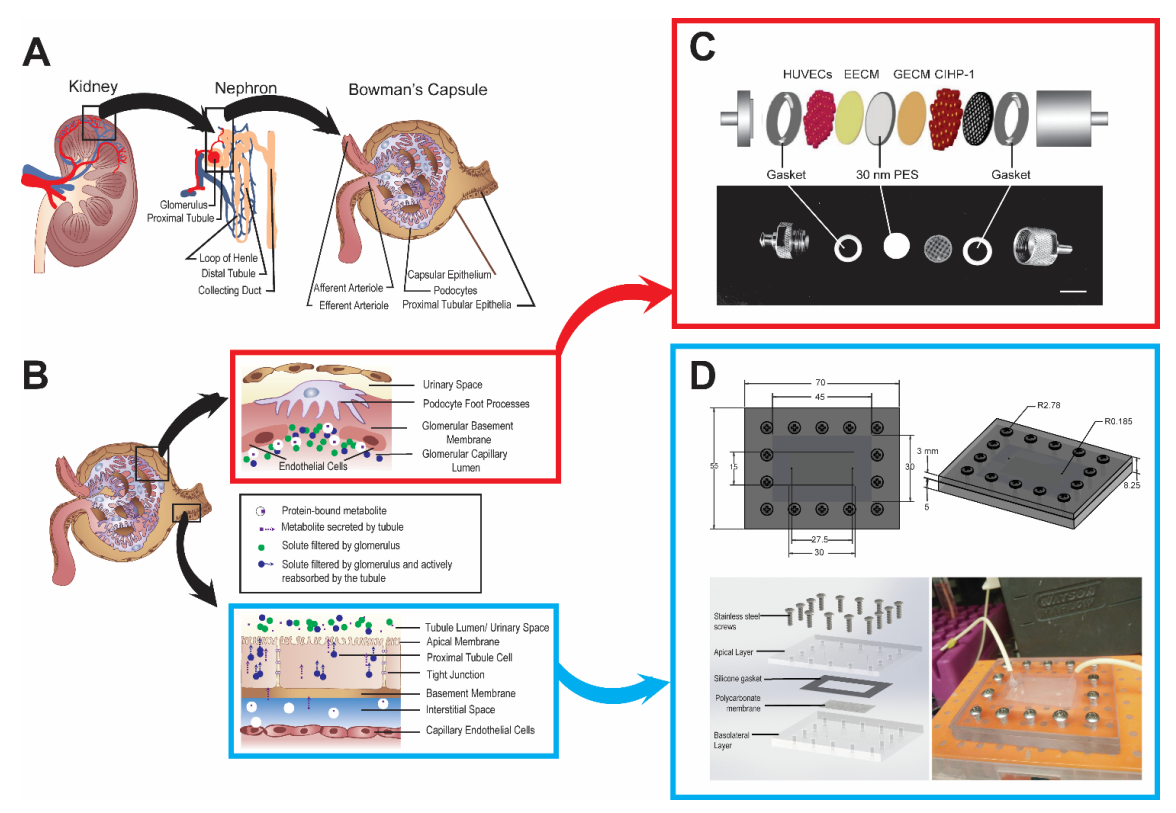


Figure 1. Translation of the Nephron into MPS. (A-B) Schematic of kidney, nephron, and its structures. (C) Schematic and image of glomerulus housing unit. Bar $=12 \mathrm{~mm}$. (D) Schematic and image of PCT device.

A

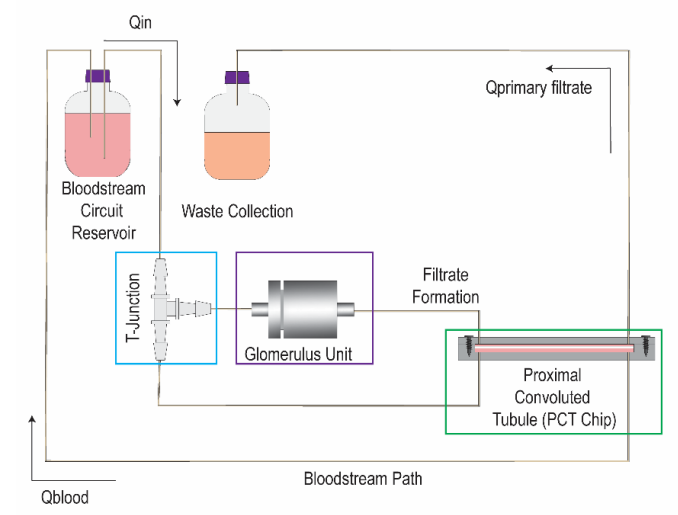

C

Differentiation of $\mathrm{CIHP}-1$

Day 0
B

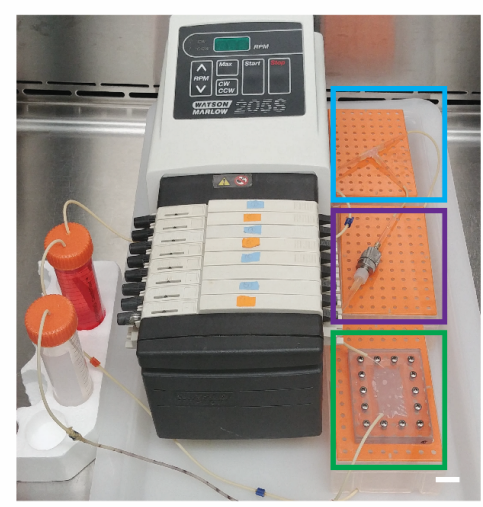

HK-2\&

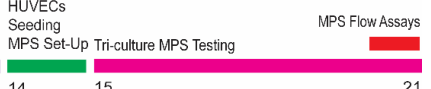

Figure 2. Construction of Glomerulus and Proximal Convoluted Tubule (PCT) MPS. (A) Schematic of MPS. (B) Image of MPS Set-Up. Bar $=15 \mathrm{~mm}$. Blue $=$ T-Junction. Purple = glomerulus housing unit. Green $=$ PCT device. (C) Timeline of MPS Experiment. 
A

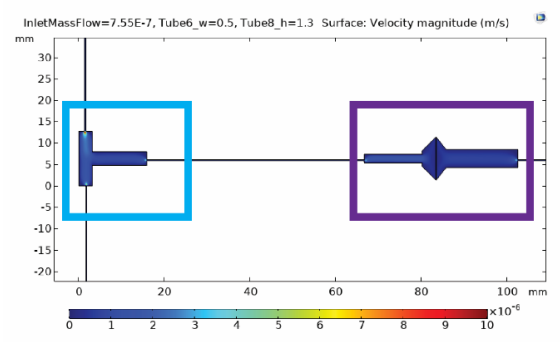

C

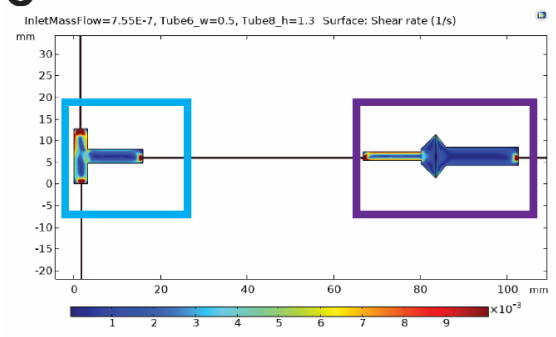

$\mathrm{E}$

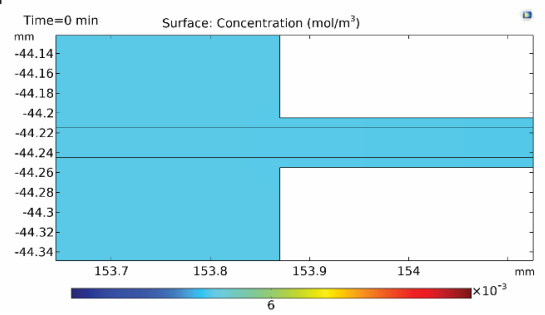

B

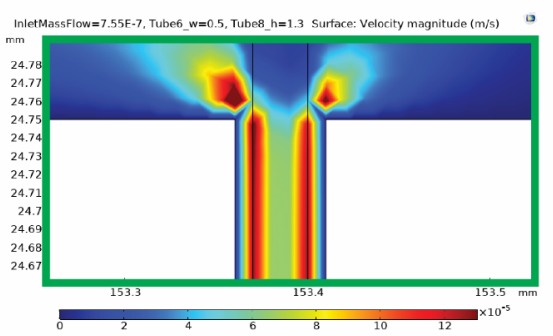

D

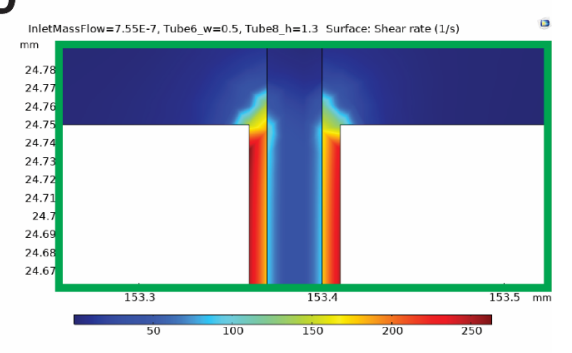

$\mathbf{F}$

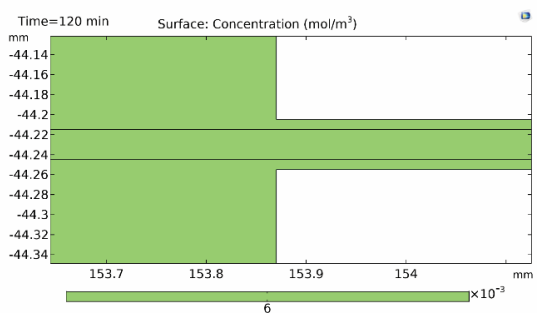

Figure 3. 2D COMSOL@ Velocity Magnitude, Shear Rates, and Concentration Profiles. (A) Velocity magnitude $(\mathrm{m} / \mathrm{s})$ in T-Junction and Glomerular Housing Unit. (B) Velocity magnitude $(\mathrm{m} / \mathrm{s})$ in proximal convoluted tubule (PCT) Device. (C) Shear Rate $\left(\mathrm{S}^{-1}\right)$ in T-Junction and Glomerular Housing Unit. (D) Shear Rate $\left(\mathrm{S}^{-1}\right)$ in PCT Device. (E) Protein concentration $(\mathrm{mg} / \mathrm{mL}$ ) at time $0 \mathrm{~min}$. (F) Protein concentration $(\mathrm{mg} / \mathrm{mL})$ at time $120 \mathrm{~min} . \mathrm{X}$ and $\mathrm{Y}$ axes $=\mathrm{XY}$ geometry coordinates $(\mathrm{mm})$. Blue $=$ T-Junction. Purple $=$ glomerulus housing unit. Green $=\mathrm{PCT}$ device. Color bar ranges from lowest (blue) to highest measurements (red), where (A) $0-10 \times 10^{-5} \mathrm{~m} / \mathrm{s}$, (B) $0-12 \times 10^{-5} \mathrm{~m} / \mathrm{s}$, (C) $0-12 \times 10^{-3} \mathrm{~S}^{-1}$, (D) 0- $250 \mathrm{~S}^{-1}$, (E) $0-6$ $\mathrm{x} 10^{-3} \mathrm{~mol} / \mathrm{m}^{3}$, (F) $0-6 \times 10^{-3} \mathrm{~mol} / \mathrm{m}^{3}$. 
A
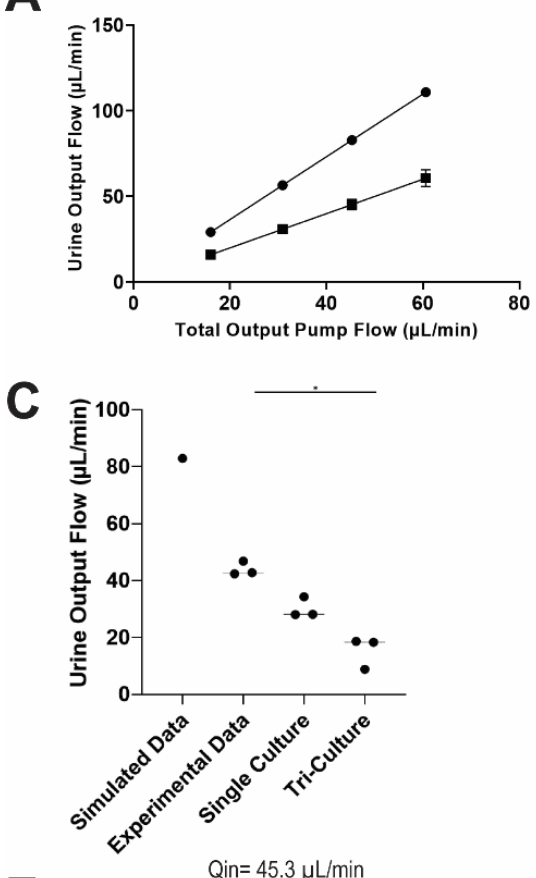

B

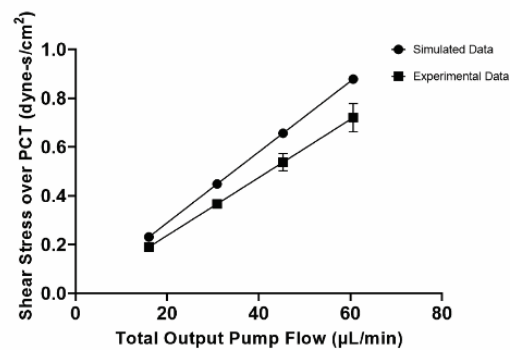

D

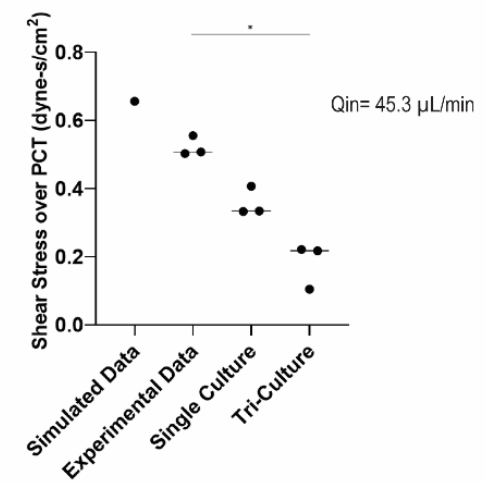

E

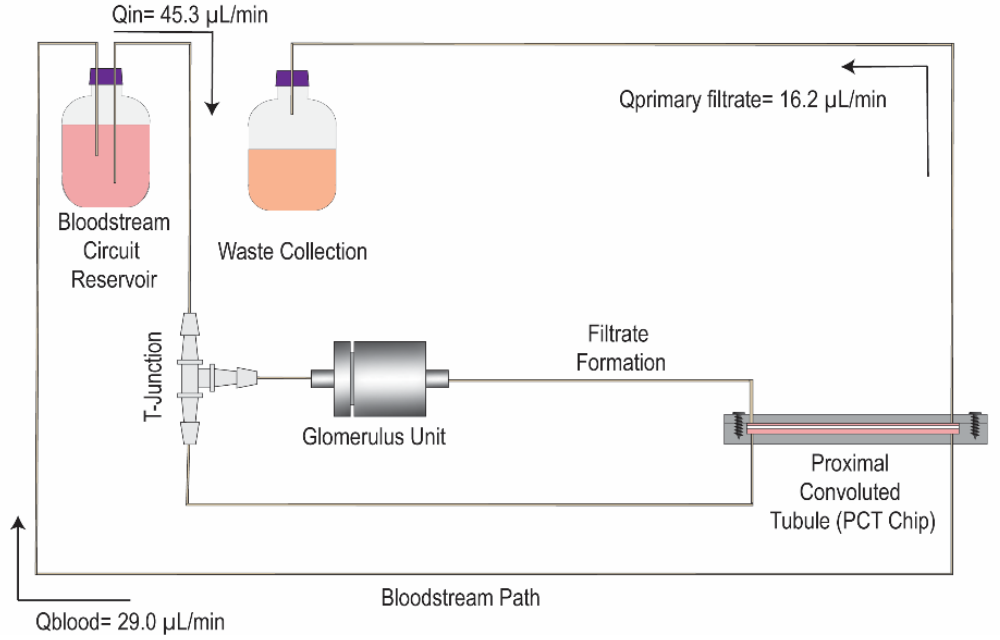

Figure 4. Simulated and Experimental Comparison of Glomerulus and Proximal Convoluted Tubule (PCT) MPS Pump Flow and Shear Stress. (A) Measured filtrate output flow $(\mu \mathrm{L} / \mathrm{min})$ for different total output pump flow $(\mu \mathrm{L} / \mathrm{min})$ in both simulated and experimental settings $\left(0.25 \mathrm{~mm}\right.$ ID tubing only). $\mathrm{R}^{2}=1.0$. (B) Shear stress across the apical PCT (dyne-s $/ \mathrm{cm}^{2}$ ) for different total output pump flow $(\mu \mathrm{L} / \mathrm{min})$ in both simulated and experimental settings $\left(0.25 \mathrm{~mm}\right.$ ID tubing only). $\mathrm{R}^{2}=1.0$. (C) Scatter plot of simulated, experimental (single inlet tubing), single-culture, and tri-culture urine output flow under $\mathrm{Q}_{\text {in }}=45.3 \mu \mathrm{L} / \mathrm{min}$. (D) Scatter plot of simulated, experimental (single inlet tubing), single-culture, and tri-culture shear stress (dyne-s $/ \mathrm{cm}^{2}$ ) under $\mathrm{Q}_{\mathrm{in}}=45.3 \mu \mathrm{L} / \mathrm{min}$. (E) Assembled MPS flow validation. Line represents median. (* Comparison of datapoints within conditional test, $\mathrm{p}<0.05$, non-parametric one-way ANOVA). 


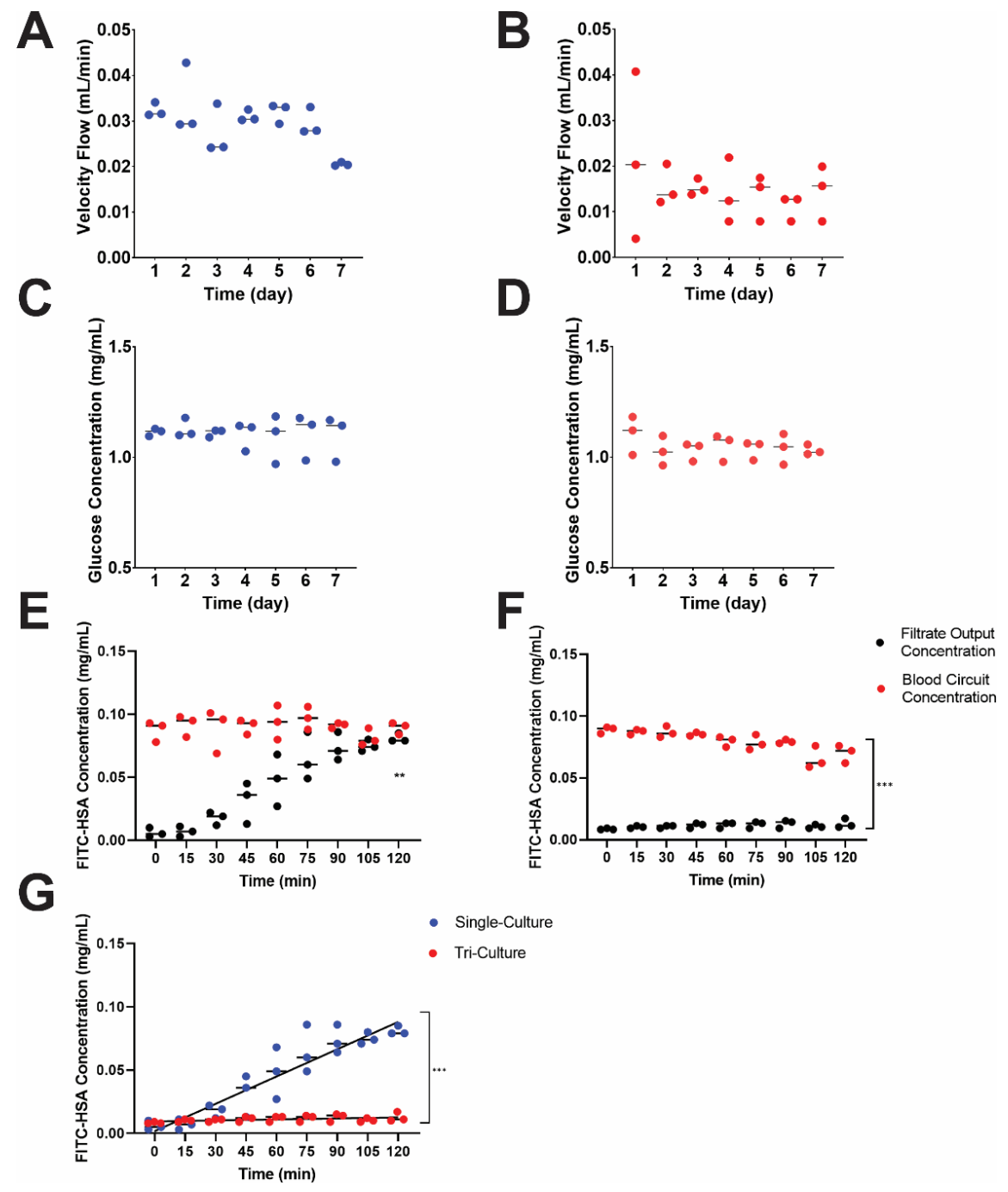

Figure 5. Functional Comparison of Glomerular and Proximal Convoluted Tubule (PCT) MPS in Single and Tri-culture Conditions ( $\mathrm{n}=3$ microsphyiological system [MPS] devices/condition). Figures A,C,E represent single culture experimental tests with no glomerular cells, and figures B, D, F depict a tri-culture MPS with a glomerulus housing unit. (A) Filtrate output formation in single culture. (B) Filtrate output formation in tri-culture. (C) Glucose secretion in single culture. (D) Glucose secretion in tri-culture. (E) Protein filtration of FITC- human serum albumin (HSA) in single culture. (** Comparison of individual datapoints within urine output, $\mathrm{p}<0.01$, non-parametric one-way ANOVA). (F) Protein filtration of FITC-HSA in tri-culture. (*** Comparison of individual datapoints within blood circuit and filtrate output, $\mathrm{p}<0.001$, non-parametric one-way ANOVA and Mann-Whitney test). (G) HSA concentration of filtrate output in single and triculture. (*** Comparison of rates in filtrate between single and tri-cultures, $\mathrm{p}<0.001$, linear regression, $\left.\mathrm{R}_{\text {single }}{ }^{2}=0.86, \mathrm{R}_{\text {tri }}{ }^{2}=0.20\right)$. 

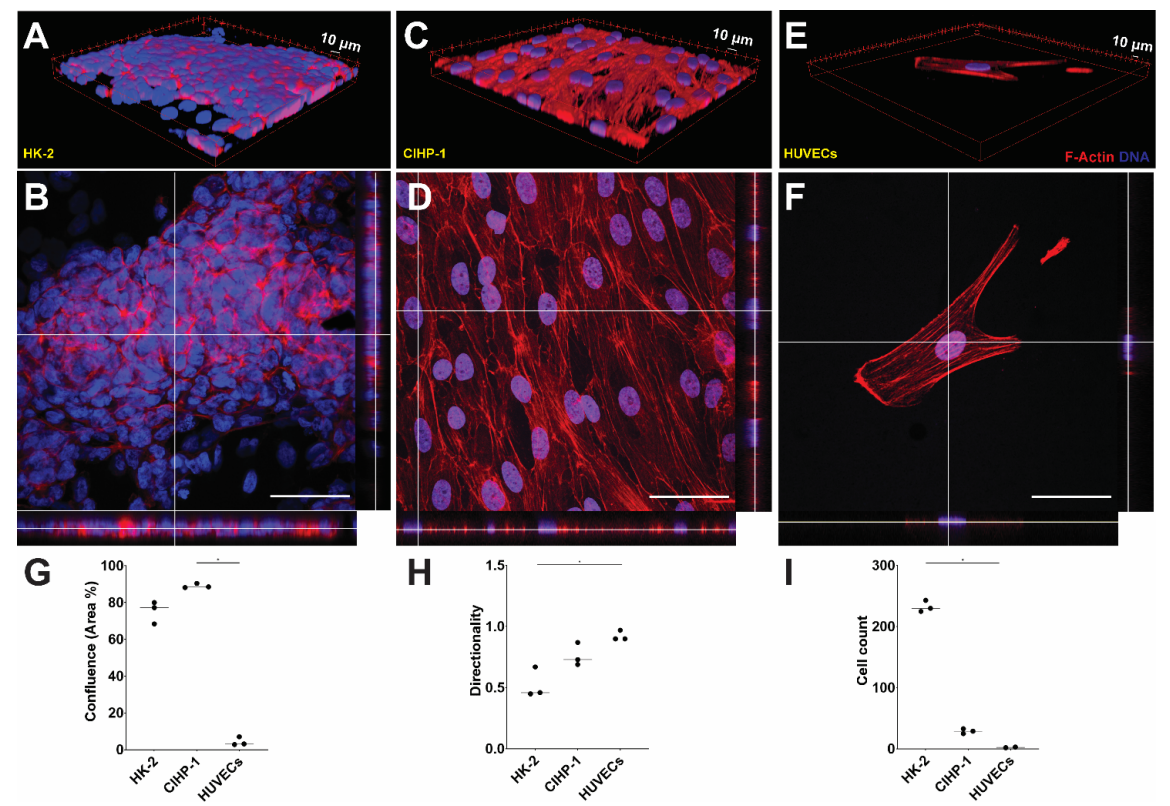

Figure 6. Post-Experiment Fluorescent-Stained Cell Images $(n=9)$. (A) 3D Projection of HK-2 on polycarbonate membrane. (B) 2D Orthogonal View of HK-2 on polycarbonate membrane. (C) 3D Projection of CIHP-1 on PES membrane. (D) 2D Orthogonal View of CIHP-1 on PES membrane. (E) 3D Projection of HUVECs on PES membrane. (F) 2D Orthogonal View of HUVECs on PES membrane. (G) Area fraction across all conditions (HK-2, CIHP-1, HUVECs). (H) Directionality across all conditions (HK-2, CIHP-1, HUVECs). (I) Cell count across all conditions (HK-2, CIHP-1, HUVECs). Red $=$ Phallodin 568, Blue= Hoescht 33342, Green = FITC-HSA. (* Comparison of individual datapoints within each cell type, $\mathrm{p}<0.05$, non-parametric one-way ANOVA).

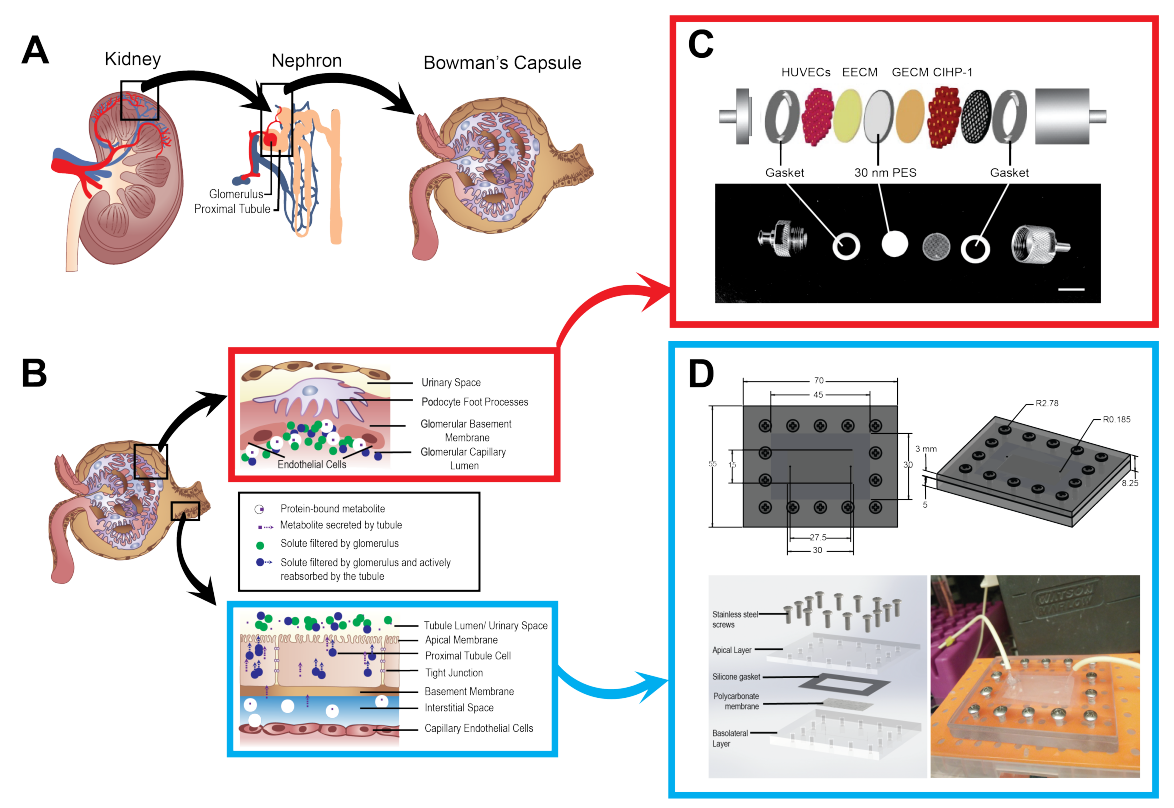


A

B
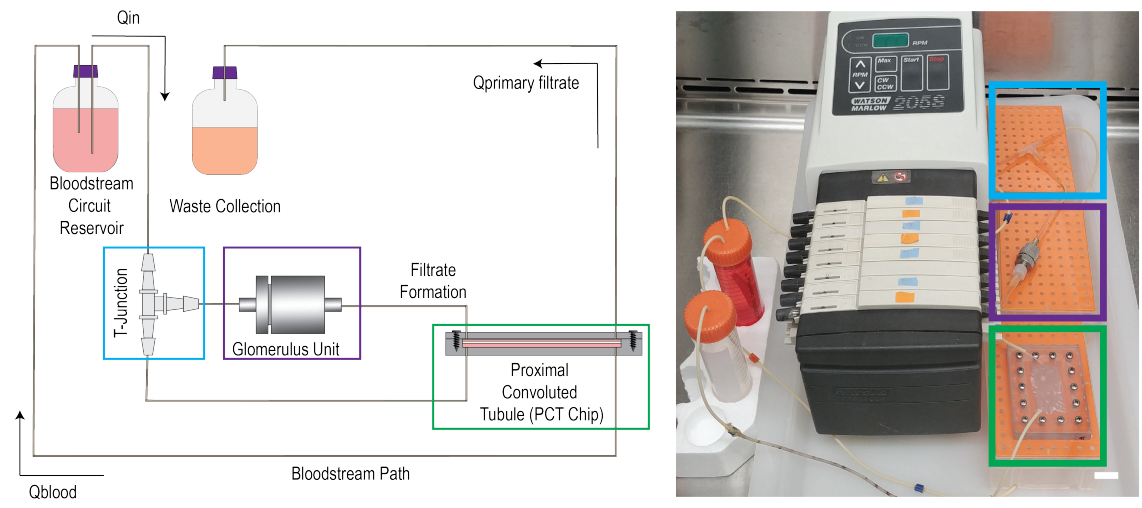

C

HK-2 \&
HUVECS

HUVECs $\quad$ MPS Flow Assays

Differentiation of CIHP-1

MPS Set-Up Tri-culture MPS Testing

Day 0

14

15

A

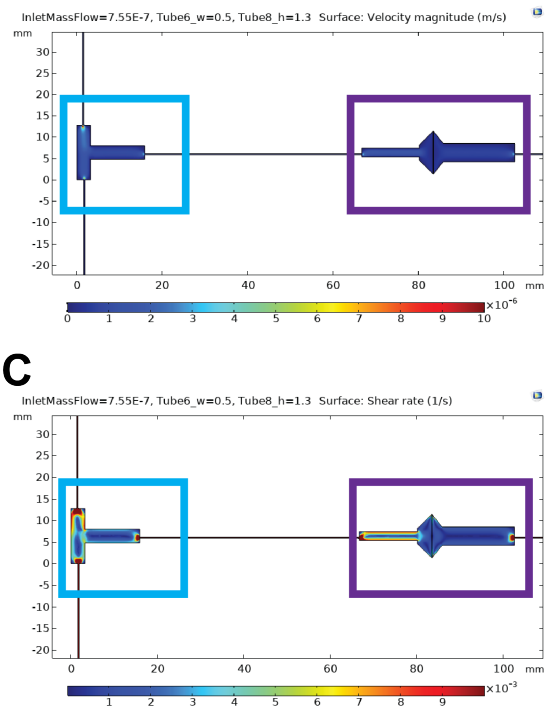

E

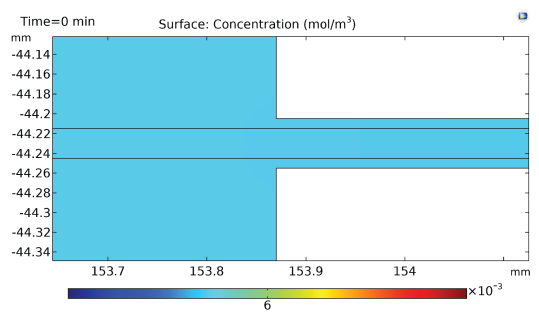

B

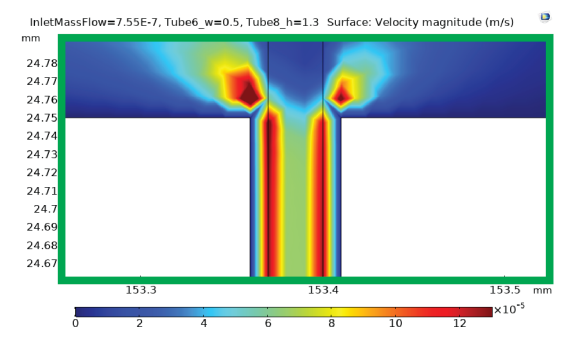

D

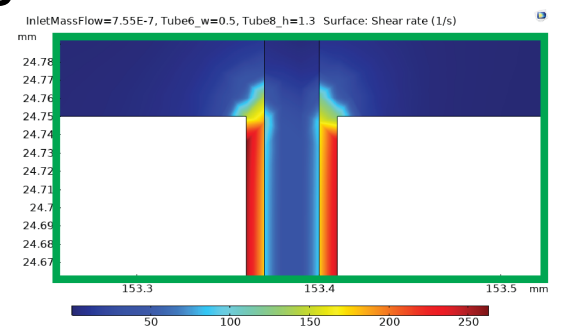

$\mathbf{F}$

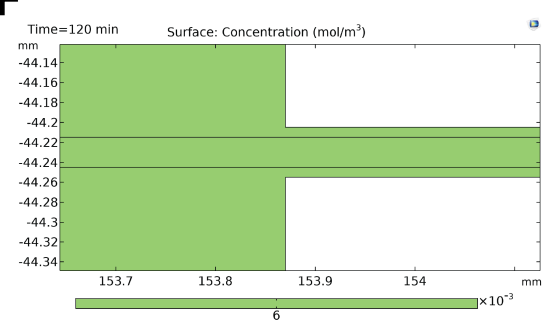


A

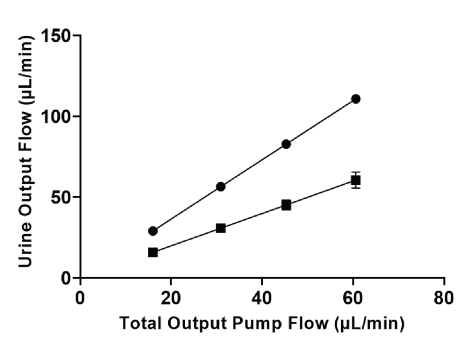

C

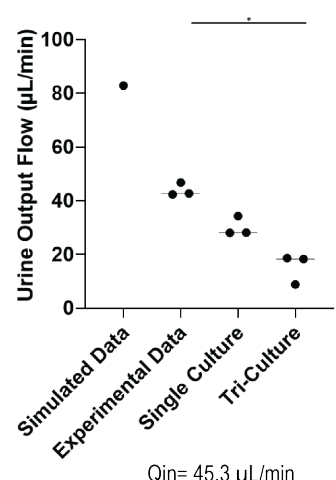

B

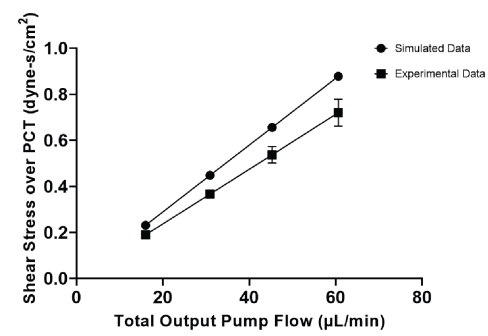

D

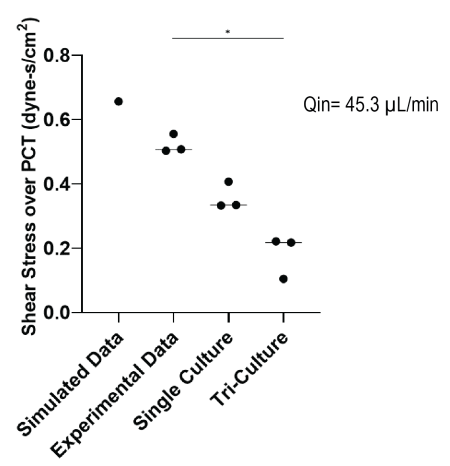

E

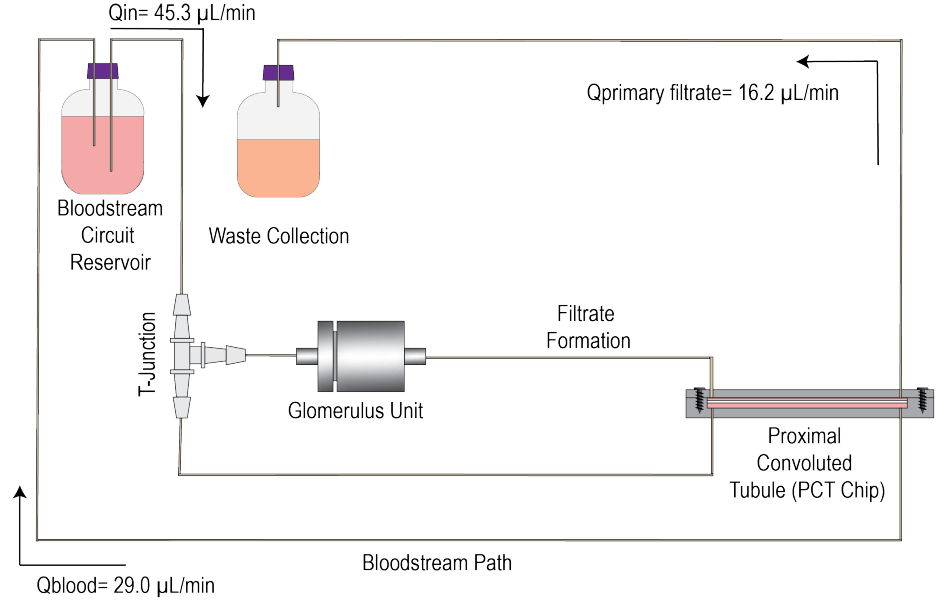




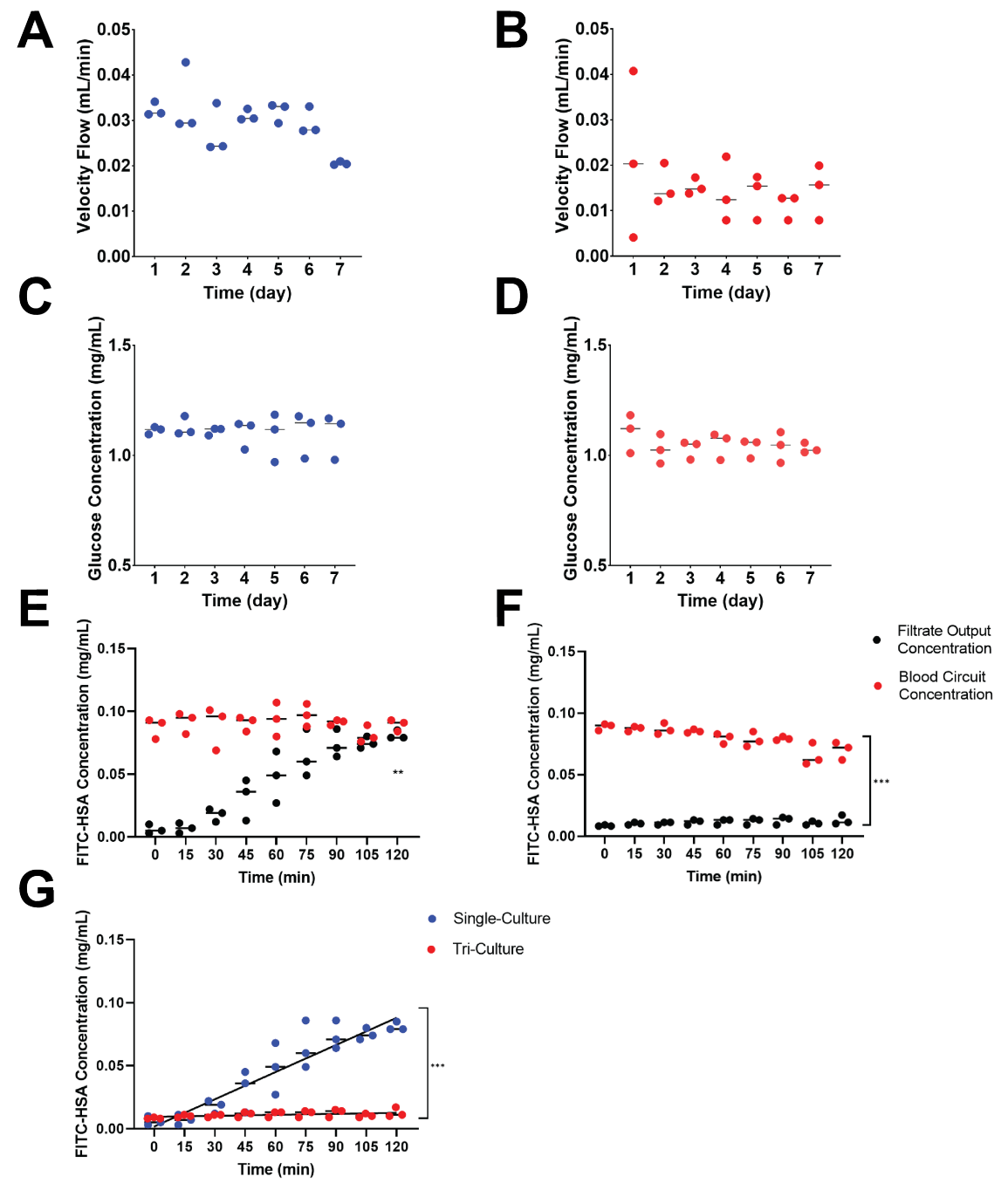



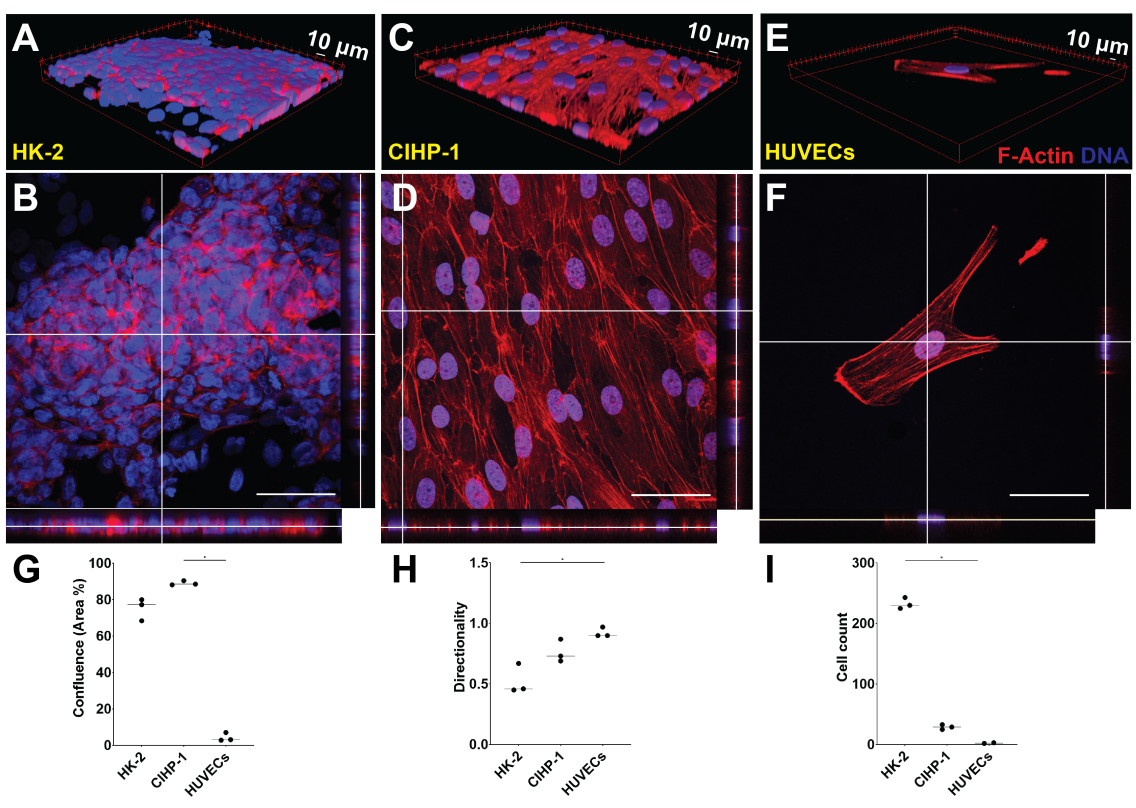

I

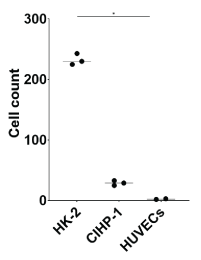

\section{Hosted file}

BnB_SZ_Table.pdf available at https://authorea.com/users/405882/articles/516711-modellingrenal-filtration-and-reabsorption-processes-in-a-human-glomerulus-and-proximal-tubulemicrophysiological-system 